\title{
The impact of microbiota on hematological disorders
}

\section{Dr. Nahed Moawad Ibrahim Rakha, MD}

\section{Lecturer of Internal Medicine, Department of Hematology and Bone Marrow transplantation, Ain Shams University}

\section{Introduction:}

The human body is host to $10^{13}$ to $10^{14}$ microbial organisms. The vast majority of these organisms are bacteria although viruses and fungi are also present at considerable amount and diversity. The gut is the main site of microbial colonization harboring a complex composition of bacteria referred to as the intestinal microbiota or sometimes as the intestinal microbiome. ${ }^{1}$

Specifying the definition of the human microbiome has been complicated by confusion about terminology: "microbiota" (the microbial taxa associated with humans) and "microbiome" (the catalog of these microbes and their genes) are often used interchangeably. ${ }^{2}$

The intestinal microbiota synthesize essential amino acids and vitamins and process otherwise indigestible components of our diet such as plant polysaccharides. A healthy and balanced intestinal microbiota also protects the host from viral and bacterial infections by preventing colonization of pathogens in the gastrointestinal tract. Imbalances in the intestinal microbiota have been linked to obesity, type 2 diabetes, irritable bowel syndrome, and

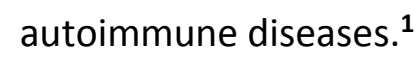

Disruption of the gut microbiome, termed dysbiosis, is frequently accompanied by overgrowth of pathogenic bacteria or fungi, in conjunction with significant loss of microbial diversity or key functional groups and an inflammatory response by the host, which contributes to disease development. Dysbiosis has been associated with an imbalance 
between populations of inflammation-mediating T-helper cells (Th1, Th2 and Th17) and antiinflammatory T-regulatory cells. ${ }^{3}$

New insights have been enabled by the development of molecular analysis methods (ie, 16S ribosomal RNA sequencing that overcome the limitations of culture-based techniques in the identification of bacterial strains. Despite a great variation in abundance of specific bacterial strains, the total set of microbial genes and the presence of genes involved in specific metabolic pathways (the metagenome) are relatively stable between healthy individuals. ${ }^{1}$

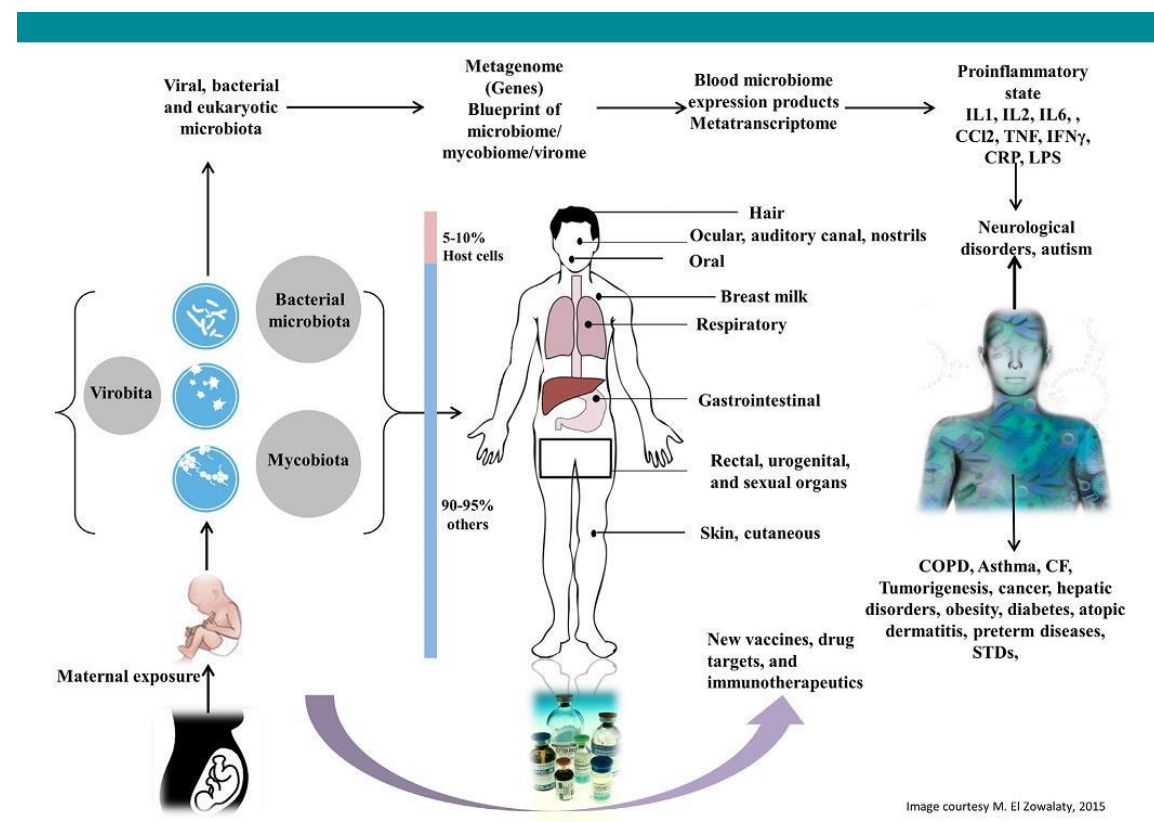

Figure (1): Schematic representation showing the human microbiota and their interplay with different body sites. The figure depicts various types of pauci-microbial including bacterial microbiota, virome, and mycobiota and their interaction with host leading to various pathological outcomes. The figure shows that virome translocates, circulates, stimulates immune system and infects cellular bacterial, fungal, and eukaryotic cells. Bottom part shows the interplay between microbiome and medicine and highlighting the advances in microbiome will help decipher new targets and discover new therapeutics.

Gut exposure to bacteria is also linked to recruitment of $T$ regulatory lymphocytes (Tregs) with the production of interleukin-10 (IL-10) and transforming growth factor- $\beta$. Moreover, intestinal bacteria are not only responsible for breaking down complex dietary fibers but are also important for the production of a variety of metabolites with active functions in the gut. The best studied of these are short-chain fatty acids (SCFAs) such as butyrate, propionate, and acetate, which serve as an energy source for intestinal epithelial cells and induce protective regulatory immune responses both locally in the gut and systemically. ${ }^{1}$ 
SCFAs serve many important roles, from regulating ion absorption and gut motility in the intestine, to modulating immune responses. SCFAs achieve these widespread functions by activating several G protein-coupled cell surface receptors, such as GPR43, which is expressed by granulocytes and some myeloid cells, and GPR109a, a receptor for niacin and C4 expressed by gut epithelial cells, adipocytes, macrophages, and dendritic cells. ${ }^{4}$

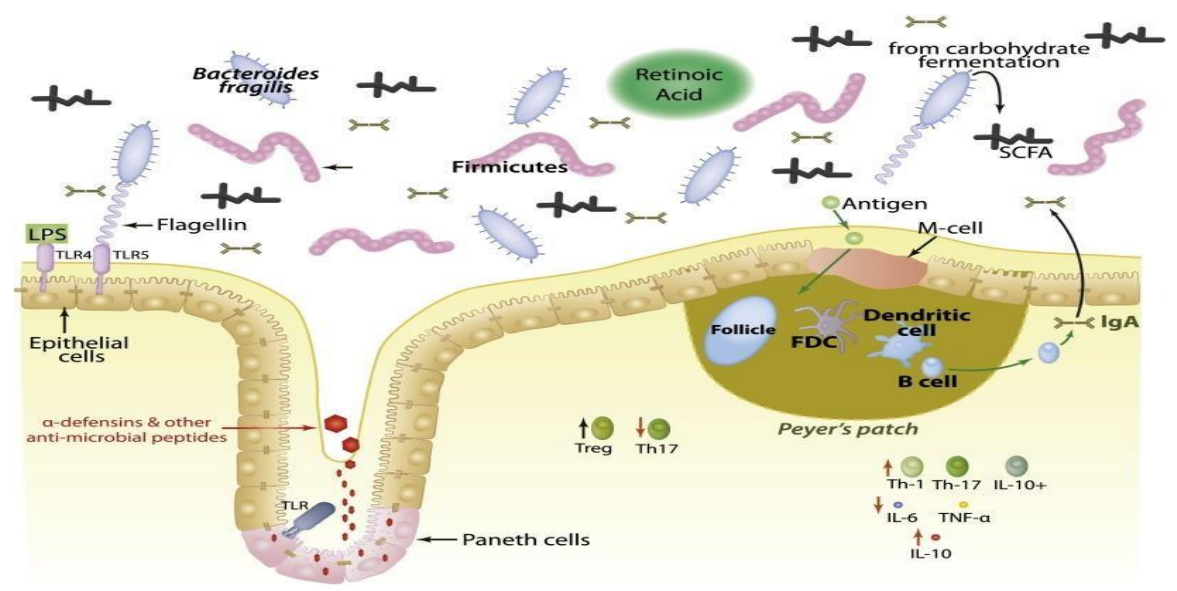

Figure (2): The role of the gut microbiota and associated products in shaping the intestinal immune system. Commensal organisms, such as bacteria in the phyla Bacteriodes and Firmicutes, colonize the gastrointestinal tract. SCFAs are produced as a result of carbohydrate fermentation, which increases the production of IL-10 and decreases the production of IL- 6 and tumor necrosis factor (TNF)- $\alpha$. Retinoic acid, which is produced by intestinal dendritic cells, leads to an increased differentiation of Tregs and decreased differentiation of inflammatory Th17 cells. The microfold (M)-cells of Peyer patches uptake antigen from the lumen and deliver it to the dendritic cells and other antigenpresenting cells located in the lamina propria. These cells become activated, and B cells secrete immunoglobulin (Ig)A into the lumen. These bacteria-specific IgA molecules thus serve to modulate the luminal microbiota composition. Paneth cells, an intestinal epithelial cell subtype that is prevalent in the small intestine and ascending colon, also shape the microbial composition by secreting $\alpha$-defensins and other antimicrobial proteins in response to bacterial antigens binding to toll-like receptors (TLRs). TLRs line the gastrointestinal tract, and microbial products such as lipopolysaccharide (LPS) and flagellin bind to TLR4 and TLR5, respectively, upregulating the expression of Regllly, a secreted antibacterial lectin that limits infection from Gram-positive bacteria. FDC, follicular dendritic cell; IL, interleukin; SCFA, short chain fatty acid; Treg, regulatory T cell; Th, helper T. ${ }^{4}$

\section{Role of microbiota in hematopoiesis}

Hematopoiesis is regulated in part by extrinsic regulators, such as growth factors and cytokines, and in part by intrinsic epigenetic and transcriptional regulators that, in concert, orchestrate differentiation of stem cells via a series of progenitor cells into all types of fully mature blood cells. ${ }^{5}$

The gut microbiota is a critical extrinsic regulator of innate and adaptive immunity as well as hematopoiesis, which ultimately maintains the vigilance of the immune system against bacterial and viral infections ${ }^{5}$. The microbiome and its associated metabolites have recently 
been functionally linked to hematopoiesis, as evidence suggests that the BM myeloid population strongly correlates with microflora complexity. ${ }^{6}$

Recent publications have been demonstrated effects of the intestinal microbiome on hematopoiesis. For instance, the intestinal microbiome, suppressible with antibiotic therapy, plays an important role in stimulating bone marrow production of granulocytes as well as influencing activation and possibly retention of granulocytes in the circulation. ${ }^{7}$

The molecular mechanisms by which commensal gut microbiota control proper immune function and hematopoiesis were recently shown to partially rely on microbial compounds such as lipopolysaccharides, which sustain steady-state production of neutrophils and their constitutive priming against bacterial infections through Toll-like receptor/myeloid differentiation factor 88-mediated signaling pathways (MyD88-mediated signaling). ${ }^{5}$

Recent reports suggested that the intestinal microbiota promote normal hematopoiesis at several stages: first by promoting activity of primitive progenitors and second by stimulating terminal granulocyte maturation. Since T cells are required for normal granulocyte maturation, a deficit in normal $\mathrm{T}$ cell activation could slow granulocyte production. ${ }^{7}$

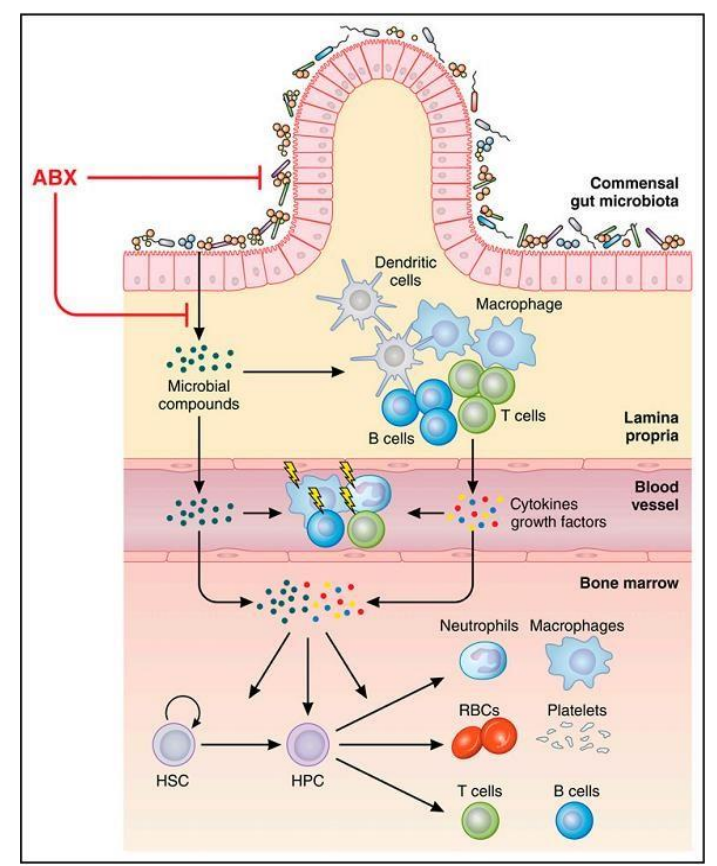

Figure [3]: Gut microbiota sustains hematopoiesis. Compounds of the commensal gut microbiota stimulate lymphocytes, macrophages, and dendritic cells in the lamina propria, which, in concert, produce a series of extrinsic stimuli. Together, these microbial and cellular stimuli maintain tonic activity of hematopoietic stem cells (HSC) and hematopoietic progenitor cells (HPC) as well as lymphocytes, monocytes, and neutrophils. Hence, microbial compounds promote steady-state hematopoiesis and vigilance of the innate and adaptive immune system against bacterial and viral infections. Broad-spectrum antibiotic treatment $(A B X)$ can disrupt the balance 
and diversity of commensal gut microbiota substantially, leading to impaired hematopoiesis and a higher susceptibility to infections. RBC, red blood cell. Professional illustration by Patrick Lane, ScEYEnce Studios 5

Given the evidence supporting a role for the microbiota in normal hematopoiesis, it is unsurprising that alterations in the microbiota, as well as in specific microbes, are associated with hematologic disorders. In some cases, pathogens are believed to trigger hematologic disorders; in others, disruption of homeostasis in the microbiota is associated with clinically significant outcomes in patients with hematologic disorders. ${ }^{4}$

\section{Links between microbiota and benign hematological disorders:}

Many anemias, such as aplastic anemia and anemia of chronic inflammation, are associated with infections and inflammatory processes, suggesting there may be an important relationship between red blood cells and the microbiota. Aplastic anemia can follow hepatitis $A, B, C, E$, and $G$ infection and is associated with parvovirus B19, cytomegalovirus (CMV), and Epstein bare virus (EBV). In anemia of chronic inflammation, inflammatory cytokines induce hepcidin expression and alter iron homeostasis. Furthermore, infections with various bacterial species have been shown to induce hepcidin. ${ }^{4}$

Primary immune thrombocytopenic purpura (ITP) remains a diagnosis of exclusion both from non-immune causes and immune thrombocytopenia that develops in the context of other disorders Immune thrombocytopenia can be secondary to medications or to a concurrent disease or chronic infection, e.g., with Helicobacter pylori, human immunodeficiency virus (HIV), or hepatitis C virus (HCV). ${ }^{8}$

Response to infection may generate antibodies that cross react with platelet antigens (HIV, $\mathrm{H}$ pylori) or immune complexes that bind to platelet Fcy receptors (HCV) and platelet production may be impaired by infection of megakaryocyte bone marrow dependent progenitor cells (HCV and HIV), decreased production of thrombopoietin, and splenic sequestration of platelets secondary to portal hypertension (HCV). ${ }^{8}$

CMV can also cause severe congenital thrombocytopenia and delayed platelet recovery after bone marrow transplantation. ${ }^{4}$

As regard to sickle cell disease, depletion of the microbiota significantly reduced the number of circulating aged neutrophils and dramatically improved the pathogenesis and inflammation-related organ damage in models of sickle-cell disease or endotoxin-induced septic shock. Microbiota regulates aged neutrophil numbers, thereby affecting both acute 
vaso-occlusive crisis and the ensuing chronic tissue damage in sickle-cell disease. ${ }^{9}$ Links between microbiota and malignant hematological disorders:

Many lymphomas are associated with the presence of specific microorganisms. Relatively strong evidence exists supporting the role of several pathogenic organisms in lymphomagenesis; these organisms include EBV, HCV, H pylori, and HIV. Lymphoma of gastric MALT type has been linked to infection with $H$ pylori. Regression of MALT lymphoma has been demonstrated in up to $70 \%$ of patients treated with antibiotics that eradicate $H$ pylori infection, suggesting a role for this common gastric microbiota resident in disease persistence. In marginal zone lymphomas, HCV is hypothesized to provide the initial antigenic stimulus for B-cell clonal expansion as part of the multistep progression toward lymphomagenesis. Other infection-lymphoma relationships have been described between the human T-cell lymphotropic virus 1 and adult T-cell leukemia/lymphoma, between EBV and endemic Burkitt lymphoma and posttransplantation lymphoproliferative disorder, and between human herpesvirus 8 and multicentric Castleman disease. ${ }^{4}$

Recent report demonstrated that cyclophosphamide alters the composition of microbiota in the small intestine and induces the translocation of selected species of Gram + ve bacteria into secondary lymphoid organs. There, these bacteria stimulate the generation of a specific subset of "pathogenic" T helper 17 ( $p$ Th17) cells and memory Th1 immune responses. ${ }^{10}$

Other report showed that disruption of the microbiota impairs the response of subcutaneous tumors to $\mathrm{CpG}$-oligonucleotide immunotherapy and platinum chemotherapy. In antibiotics-treated or germ-free mice, tumor-infiltrating myeloid-derived cells responded poorly to therapy, resulting in lower cytokine production and tumor necrosis after CpGoligonucleotide treatment and deficient production of reactive oxygen species and cytotoxicity after chemotherapy. Thus, optimal responses to cancer therapy require an intact commensal microbiota that mediates its effects by modulating myeloid-derived cell functions in the tumor microenvironment. These findings underscore the importance of the microbiota in the outcome of disease treatment. ${ }^{11}$

\section{Microbiota and hematopiotic stem cell transplantation:}

Hematopoietic stem cell transplantation (HSCT) is a potentially life-saving therapy for patients with both malignant and nonmalignant hematologic disorders. Transplantation, however, comes at the cost of potential complications from chemotherapy and radiation, graft-versus-host disease (GVHD), and post-transplant infections. Gastrointestinal (GI) toxicity 
is common after HSCT, with up to $50 \%$ of transplant recipients experiencing clinically significant diarrhea. An increasing awareness of the contribution of the environment to these disease phenotypes is being explored. One such potentially modifiable component of the environment is the gut microbiota, and this is increasingly being explored. ${ }^{\mathbf{1 2}}$

The gut microbiota and its metabolites have been reported to play pivotal roles in intestinal inflammation and the immune system. Also in allo-SCT, increasing evidence indicates that the gut microbiota is closely associated with aGVHD. ${ }^{13}$

Under normal conditions, commensal bacteria produce antimicrobial peptides to maintain the compositional diversity and immunomodulatory function of the gut microbiota.

Disturbance in the microbiota composition could occur during the process of allo-BMT that comprises bone marrow ablation by radiotherapy or chemotherapy, antibiotics treatment, and damage in mucosal barrier due to GVHD. Evidence suggested that microbiota diversity is an independent determinant of the incidence of GVHD following allo-BMT. ${ }^{14}$

The intestinal injury caused by the conditioning regimen results in increased intestinal permeability that permits translocation of gut bacteria over the intestinal barrier, a condition described as leaky bowel syndrome. As a consequence, immune stimulation from a range of pathogen and danger-associated molecular patterns such as lipopolysaccharide and peptidoglycan reinforce the inflammatory cytokine response and provide the ideal setting for T-cell alloactivation and priming. In line with this, the Toll-like receptors for these molecules have been linked to GVHD development. ${ }^{1}$

Recent studies revealed that gut colonization by antibiotic-resistant bacteria decreases the overall survival of patients undergoing alloSCT by increasing non relapse mortality and the incidences of systemic infection and aGVHD. ${ }^{15}$ Also selecting antibiotics with a more limited spectrum of activity (especially against anaerobes) could prevent microbiota injury and thus reduce GVHD in the colon and GVHD-associated mortality. ${ }^{16}$

Some investigators are exploring alternative antibiotic prophylactic strategies that may better preserve patient flora and maintain gut homeostasis. Rifaximin is a broadspectrum antibiotic with negligible intestinal absorption used clinically to treat irritable bowel syndrome and traveler's diarrhea. The use of rifaximin in HCT patients has been found to preserve intestinal diversity and associates with lower treatment-related mortality rates compared with patients treated with a combination of ciprofloxacin and metronidazole in a retrospective study. ${ }^{1}$ 
A dramatic shift in the major composition of microbiota occurs with the onset of GVHD. Normal gut microbiota consists of mainly Bacteroidetes and Firmicutes. Firmicutes comprises more than $80 \%$ of stool microbiome. Among the phylum Firmicutes, Clostridia (Clostridiumand Eubacterum) constitutes the majority of the normal microflora, but Bacilli (Lactobacillusand Enterococcus) increases dramatically in GVHD. It is also suggested that if a relative abundance of Enterococcus, which is almost absent in normal microflora, exceeds $20 \%$ of total microbiota in patient's stool specimen, the risk of GVHD frequency increases. ${ }^{14}$

A decrease in anaerobic bacteria and, in particular, the genus Blautia through antibiosis was associated with an increased incidence of GVHD in those undergoing allogeneic HSCT. $^{12}$

Efforts to understand the potential mechanistic link between loss of microbiota diversity and poor outcomes will also be informative. One hypothesis linking dysbiosis and mortality is that these dramatic alterations of the intestinal microbiota, in conjunction with neutropenia and mucosal barrier injury, can lead to complications such as septicemia. Alternatively, given the strong link between microbiota diversity and the generation of immunologic diversity in the setting of development, another hypothesis is that low microbiota diversity may impact the robustness and speed of immune reconstitution. ${ }^{4}$

Several immunologic cell types play a critical role in modulating and responding to the gut microbiota. Antigens from colonic commensal microbiota are important in educating Tregs. T-regs and IL-10-producing type 1 regulatory T-like cells compose the major regulatory populations in the intestine. Paneth cells, which are located next to intestinal stem cells within the crypts of the intestinal lumen, secrete antimicrobial peptides and $\alpha$-defensins. $\alpha$ Defensins are active against many Gram-negative and Gram-positive bacteria, fungi, and enveloped viruses but are typically selectively active against non-commensals. These molecules are hypothesized to function largely by forming pores in bacterial cell walls or by disrupting the cell membrane. In GVHD, for example, Paneth cells are lost, resulting in decreased expression of $\alpha$-defensins, which can lead to an expansion of non-commensal bacteria and decreased microbial diversity. ${ }^{4}$ 


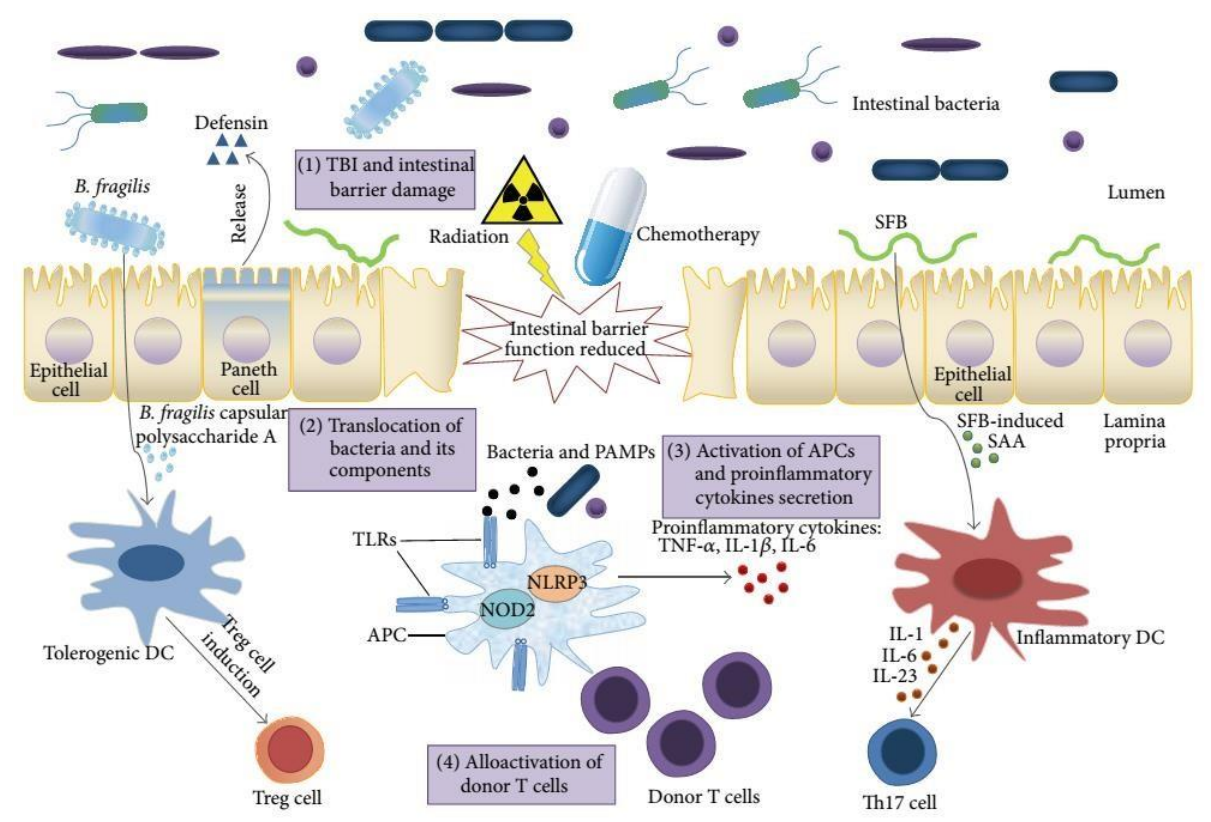

Figure [5]: Schematic of the role of microbiota in graft-versus-host diseasein the gut. Under normal conditions, the intestinal epithelial surface maintains an intact barrier function that prevents bacterial invasion into deeper host tissues. Paneth cells secrete several microbicidal proteins, including-defensins, which selectively kill pathogenic bacteria.Bacteroides fragilis-derived TLR capsular polysaccharide A can promote the induction of Treg cells. Segmented filamentous bacteria (SFB) induce production of serum amyloid A (SAA) in the gut, and SAA acts on dendritic cells (DCs) to promote Th17 cell differentiation. After total body irradiation (TBI) and chemotherapy as part of the conditioning regimen, the integrity of the intestinal surface is decreased. Intestinal bacteria and their components (pathogen-associated molecular patterns, PAMPs) translocate to the lamina propria and are recognized by Toll-like receptors (TLRs) in host antigen-presenting cells (APCs). Activated APCs secrete proinflammatory cytokines and prime donor T cells, which aggravate acute GVHD ${ }^{17}$

With improved technology to understand the ecosystem of microorganisms that make up the gut microbiota, there is increasing evidence of the microbiota's contribution to the development of post-transplant complications. Antibiotics have traditionally been the mainstay of microbiota altering therapies available to physicians. Recently, interest is increasing in the use of prebiotics and probiotics to support the development and sustainability of a healthier microbiota. ${ }^{12}$

Accumulating evidence suggest that several strategies could be trialed in steps of alloBMT to maintain microbiota diversity and prevent enterococcal domination. Thus, examination of patient's stool specimen could be performed to determine the timing for alloBMT, especially for the patients who underwent antibiotics administrations. Alternatively, modulation of gut microbiota by introducing beneficial commensal bacteria could be considered to improve the medical prognosis. ${ }^{14}$

Prevention of hospital acquired Enterococcus infections could be attained from the hospital care units. Maintaining near sterile environment has to be achieved as the first line 
of defense against E. faecalis and/or E. faecium infections. Hospitalization of patients in a laminar air flow room before and after allo-BMT could be considered and a strict hygienic management has to be performed. Given broad spectrum antibiotic administration increases vancomycin resistant Enterococcus susceptibility, selection of antibiotics has to be practiced with caution. Further identification of genera involved in GVHD related morality followed by a re-affirmation across BMT centers would increase our understanding and support a development of therapeutics to modulate gut microbiota.$^{14}$

Overall, microbiota-based therapeutics hold great promise for the prevention and treatment of GVHD and infections in HSCT patients. Significant opportunity exists for further research into the development of targeted and individualized dysbiosis prevention and treatment regimens applicable to HSCT patients. ${ }^{12}$

\section{Benefits behind microbiota in hematological field:}

Tools and approaches to modify the microbiota are also being developed in the hope of improving health outcomes. Interventions range from biological therapies such as fecal microbiota transfer (FMT), to modification of the diet, to personalized and targeted antibiotic therapies designed to tailor the exact composition of the microbiota. ${ }^{4}$

FMT refers to infusion of a fecal suspension from a healthy donor into the gastrointestinal tract of a patient to restore a healthy microbiota and cure disease. Manipulation of the intestinal microbiota by FMT may influence the immune system and improve immune-mediated enteritis such as gut aGVHD especially corticosteroid resistance cases. However, FMT carries a potential risk of infection by the infused microbiota for SCT recipients. ${ }^{14}$

FMT can be performed via the introduction of stool or a purified bacterial stool product via nasogastric tube, colonoscopy, or capsulized therapy. This technique has been used to successfully treat recurrent Clostridium difficile-associated disease. FMT results in a change in bacterial composition that is accompanied by resolution of patient symptoms. ${ }^{4}$ Interestingly it was found, in a preliminary study, that FMT was able to eradicate resistant bacteria harbored in the gut of an immunocompromised patient affected by multiple myeloma. ${ }^{18}$

Increasing attention has been given to the potential of probiotics and prebiotics to prevent and treat intestinal dysbiosis. Probiotics are nutritional supplements containing a defined amount of viable microorganisms that upon administration confer a benefit to the 
host. ${ }^{1}$ The most commonly used probiotic organisms \{Lactobacillus spp. and Bifidobacterium spp.\} are consumed in large quantities as part of traditional foods across the globe. ${ }^{12}$ Whereas prebiotics consist of non-digestible food ingredients (eg, undigested fibers) that support the growth of beneficial bacteria. Only a few studies have addressed the feasibility of prebiotic or probiotic treatment in $\mathrm{HCT}$, and there will be major safety issues involved in administering viable bacteria to severely immune-compromised HCT patients. ${ }^{1}$

Some of the beneficial impacts of probiotics have been scientifically documented, and three main categories of probiotics contributing to host health have been described. First, certain probiotics can exclude or inhibit pathogens. Second, probiotics can enhance the function of the intestinal epithelial barrier by modulating various signaling pathways, inducing mucus and antimicrobial peptide production, enhancing tight junction functioning, and preventing apoptosis. Third, probiotics can modulate host immune responses, resulting in both local and systemic effects. ${ }^{17}$

In the future, we may be able to use increasing knowledge of the microbiota to develop precision prebiotics that are even more powerful in expanding commensal flora or that influence organisms towards a particular shift of the gastrointestinal metabolome. Probiotics could potentially be engineered to support the gut microbiota while also having a biological off-switch that could be triggered at the first suspicion of bacteremia. The development of designer microbiota with non-pathogenic organisms may be of use even in highly immunocompromised patients undergoing HSCT. An early detection of dysbiosis could be treated with an individualized microbiota transfer of commensal organisms necessary to enhance diversity for the prevention of subsequent bacteremia and sepsis. ${ }^{12}$

\section{References:}

1. Staffas A, da Silva MB, van den Brink M. The intestinal microbiota in allogeneic hematopoietic cell transplant and graft-versus-host disease. Blood. 2017; 129(8): 927-933.

2. Ursell LK, Metcalf JL, Parfrey LW, et al. Defining the Human Microbiome. Nutr Rev. 2012; 70(Suppl 1): S38S44.

3. Fujimura KE, Slusher NA, Cabana MD, et al. Role of the gut microbiota in defining human health. Expert Rev Anti Infect Ther. 2010; 8(4): 435-454.

4. Manzo VE, Bhatt AS. The human microbiome in hematopoiesis and hematologic disorders. Blood. 2015; 126(3): 311-318.

5. Kim Theilgaard-M“onch. Gut microbiota sustains hematopoiesis. Blood. 2017; 129 (6): 662-663. 
6. Espinoza JL, Kotecha R, Nakao S. Microbe-induced inflammatory Signals Triggering Acquired Bone Marrow Failure Syndromes. Front. Immunol. 2017; 8(186):1-8.

7. Josefsdottir KS, Baldridge MT, Kadmon CS, et al. Antibiotics impair murine hematopoiesis by depleting intestinal microbiota. Blood. 2016; 129(6): 729-739.

8. Cines DB, Liebman H, Stasi R. Pathobiology of secondary immune thrombocytopenia. Semin Hematol. 2009; 46(1 Suppl 2): S2-14 (abstract).

9. Zhang D, Chen G, Manwani D, et al. Neutrophil ageing is regulated by the microbiome. Nature. 2015; 525: 528-532.

10. Viaud S, Saccheri F, Mignot G, et al. The intestinal microbiota modulates the anticancer immune effects of cyclophosphamide. Science. 2013; 342(6161): 971-976.

11. lida N, Dzutsev A, Stewart CA, et al. Commensal bacteria control cancer response to therapy by modulating the tumor microenvironment. Science. 2013; 342(6161): 967-970.

12. Andermann TM, Rezvani A,\& Bhatt AS. Microbiota Manipulation With Prebiotics and Probiotics in Patients Undergoing Stem Cell Transplantation. Curr Hematol Malig Rep. 2016; 11:19-28.

13. Kakihana K, Fujioka Y, Suda W, et al. Fecal microbiota transplantation for patients with steroid-resistant acute graft-versus-host disease of the gut. Blood. 2016; 128(16):2083.

14. Yu-Hee Kim. Gut Microbiota in Graft-versus-Host Disease. Journal of Bacteriology and Virology. 2016; 46(4): $307-311$.

15. Bilinski J, Katarzyna Robak K, Peric Z, et al. Impact of Gut Colonization by Antibiotic-Resistant Bacteria on the Outcomes of Allogeneic Hematopoietic Stem Cell Transplantation: A Retrospective, Single-Center Study. Biol Blood Marrow Transplant. 2016; 1-7.

16. Shono Y, Docampo YD, Peled JU, et al. Increased GVHD-related mortality with broad-spectrum antibiotic use after allogeneic hematopoietic stem cell transplantation in human patients and mice. Sci Transl Med. 2016; 8(339): 339-71.

17. Chen Y, Zhao Y, Cheng Q, et al. The Role of Intestinal Microbiota in Acute Graft-versus-Host Disease. Journal of Immunology Research. 2015; 1-9.

18. Laterza L, Rizzatti G, Gaetani E, et al. The gut microbiota and immune system relationship in human graft versus-host disease. Mediterr J Hematol Infect Dis. 2016; 8(1): 1-10. 